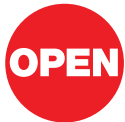

SUBJECT AREAS:

GLASSES

SURFACES, INTERFACES AND

THIN FILMS

STRUCTURE OF SOLIDS AND

LIQUIDS

COLLOIDS

Received

6 November 2012

Accepted

27 December 2012

Published

15 January 2013

Correspondence and requests for materials should be addressed to P.S. (ps@peterschall.

de)

\section{Probing incipient plasticity by indenting colloidal glasses}

\author{
Y. Rahmani, R. Koopman, D. Denisov \& P. Schall
}

Institute of Physics, University of Amsterdam, Science Park 904, Amsterdam, the Netherlands.

Glasses are lucrative engineering materials owing to their superior mechanical properties such as high strength and large elastic strain. A central question concerns incipient plasticity - the onset of permanent deformation - that is central to their relaxation, aging, yield and fracture. Here, we use an analogue of nano-indentation performed on a colloidal glass to obtain direct images of the incipient plasticity, allowing us to elucidate the onset of permanent deformation. We visualize the microscopic strain by following distorted nearest neighbor configurations, and observe a surprising hierarchical structure of deformation: at the onset of irreversible deformation, the strain acquires a robust fractal structure, and we measure its fractal dimension. These results give direct evidence that the onset of permanent deformation has the hallmarks of a critical point, in agreement with recent theoretical works.

ncipient plasticity addresses a crucial step in the deformation of materials. It marks the onset of permanent deformation due to irreversible atomic rearrangements. At small applied strain and short times, solids deform elastically, being able to recover their shape when the strain is removed. However, at larger applied strain and on longer time scales, irreversible atomic rearrangements occur, leading to permanent deformation. Glasses are frozen on experimental time scales, and the onset of irreversible rearrangements is crucial for their relaxation, aging and deformation ${ }^{1-3}$. Nanoindentation experiments can provide crucial insight into the incipient plastic deformation by resolving individual relaxation events that govern the very earliest stages of plastic deformation ${ }^{4-6}$. While nanoindentation experiments on conventional materials allow the resolution of individual elementary plastic events from force-displacement measurements ${ }^{7,8}$, however, the direct imaging of the elementary relaxation, and of the microscopic displacements at the onset of plastic deformation is prohibitively difficult. Colloidal glasses provide good models for visualizing the structure and dynamics of glasses in real space. Here, we use an analogue of nanoindentation on a colloidal glass to obtain direct images of the incipient deformation. We track the individual particles in three dimensions, and directly follow the microscopic strain during the indentation, allowing us to elucidate the nature of the incipient deformation. We find robust scaling relations of the microscopic strain, giving direct evidence that the onset of permanent deformation has the hallmarks of a critical point, in agreement with recent theoretical work on fibrous networks'. Such critical behavior has important consequences for the application of amorphous materials as new engineering materials at small scales ${ }^{1}$.

\section{Results}

Dense colloidal suspensions provide good models to elucidate deformation at small scales; at high density, the motion of the particles becomes arrested similar to the atomic motion in conventional glasses. We prepared a dense amorphous colloidal sediment roughly 40 particle diameters thick, and used a sewing needle that we push slowly into the sediment to indent the colloidal glass (see Methods). The length scale ratio of the needle diameter, the colloidal particle size and the film thickness are similar to those used in nano-indentation experiments; hence, the experiments are comparable. The needle touches the surface of the sediment at time $t=0$. The colloidal model allows us to follow individual particle displacements deep in the bulk of the colloidal glass. We show particle displacements after 21 min of indentation in Fig. 1b. Large displacements concentrate below the tip as expected; we find that, however, even far away from the tip, pronounced displacements occur. To explore the deformation of the glass in more detail, we determine the local strain from distorted nearest neighbor configurations. Nearest neighbors of a particle are determined as those separated by less than $r_{1}$, the first minimum of the pair correlation function. Equally, one could determine nearest neighbors from the Voronoi tessellation of space. We find that the result does not depend on the particular choice of nearest neighbors. For each particle, we then follow its nearest neighbors in time, and determine the best affine deformation tensor $\alpha$ that transforms the change of nearest neighbor vectors over the time interval, $\Delta t$. To do so, the best affine transformation tensor that transforms the 

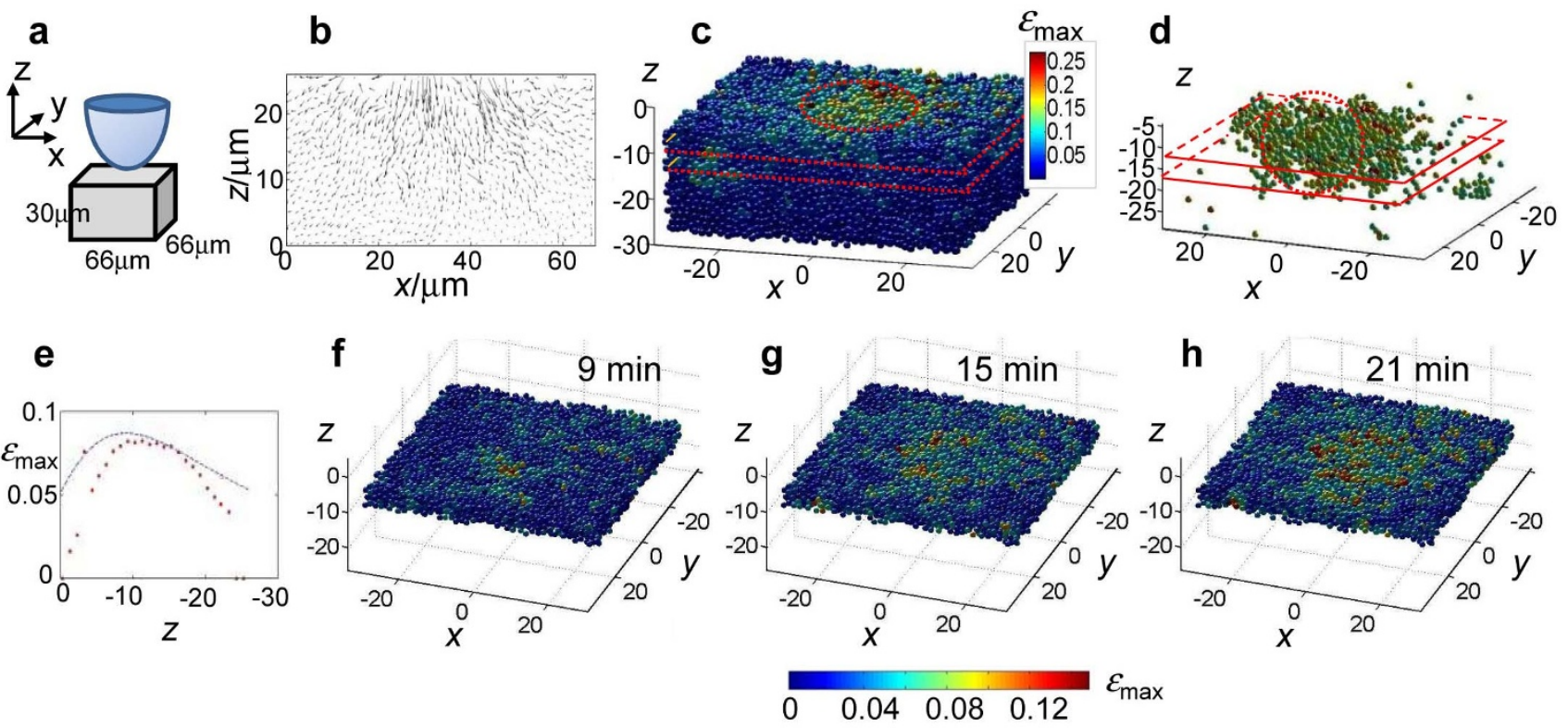

$\begin{array}{llll}0 & 0.04 & 0.08 & 0.12\end{array}$

Figure $1 \mid$ Indentation setup and strain distribution. (a) Schematic showing the needle tip with respect to the amorphous colloidal film, and the imaged $66 \mu \mathrm{m}$ by $66 \mu \mathrm{m}$ by $30 \mu \mathrm{m}$ volume. (b-d) Particle displacements and strain distribution after 23 min of indentation. (b) Particle displacements in a $5 \mu \mathrm{m}$ thick section centered below the needle. Arrows are enlarged by a factor of two for clarity. (c, d) $66 \mu \mathrm{m}$ by $66 \mu \mathrm{m}$ by $25 \mu \mathrm{m}$ reconstruction of the strain distribution. Particle color indicates the magnitude of the maximum local shear strain, $\varepsilon_{\max }$ (see color bar). In (d), particles with $\varepsilon_{\max }<0.1$ have been omitted for clarity. (e) Maximum shear strain as a function of height below the tip, experimental measurement (red dots) and continuum elasticity prediction (dashed curve). (f)-(h) Time series showing the evolution of the maximum shear strain in a $66 \mu \mathrm{m}$ by $66 \mu \mathrm{m}$ by $5 \mu \mathrm{m}$ section, $10 \mu \mathrm{m}$ below the needle, after $9 \min (\mathrm{f}), 15 \mathrm{~min}(\mathrm{~g})$, and $21 \mathrm{~min}(\mathrm{~h})$ of indentation.

nearest neighbor vectors $\boldsymbol{d}_{i}(t)$ at time $t$, to the vectors $\boldsymbol{d}_{i}(t+\Delta t)$ at time $t+\Delta t$ is determined by minimizing the mean square difference $\sum_{i}\left(\boldsymbol{d}_{\boldsymbol{i}}(t+\Delta t)-\alpha \boldsymbol{d}_{\boldsymbol{i}}(t)\right)^{2}{ }^{10}$. The symmetric part of $\alpha$ corresponds to the local strain tensor $\varepsilon_{i j}$ of the particle under consideration. A good measure of the local shear deformation is the maximum shear strain, $\varepsilon_{\max }=\left|\varepsilon_{1}-\varepsilon_{2}\right| / 2^{11}$, an invariant of the strain tensor, where, $\varepsilon_{1}$ and $\varepsilon_{2}$ are the largest and smallest eigenvalues of the strain tensor. The maximum shear strain $\varepsilon_{\max }$ reflects the shear strain acting along the principal axes of the strain tensor, and has been used as a good measure of strain in the inhomogeneous deformation of isotropic materials ${ }^{11}$. To visualize its distribution, for each particle, we determine values of $\varepsilon_{\max }$ from the eigenvalues of the strain tensor associated with the particle. Reconstructions of the resulting strain distributions are shown in Fig. $1 \mathrm{c}$ and d. Orange and yellow spheres indicate particles with large $\varepsilon_{\max }$, while blue particles indicate small $\varepsilon_{\max }$. In Fig. 1d, we have omitted particles with $\varepsilon_{\max }<0.11$ for clarity. Orange particles accumulate below the tip, indicating strong shear strain. A strain maximum occurs at roughly $10 \mu \mathrm{m}$ below the tip (Fig. 1e) in good agreement with continuum theory predictions. In contrast to continuum elasticity predictions, however, large shear strain occurs surprisingly far away from the tip. To demonstrate this, we focus on a horizontal section, $10 \mu \mathrm{m}$ below the tip, and show the time evolution of strain in Figs. If to h (see supplementary video). Already at early times (Fig. If and g), large shear strain occurs throughout the field of view as indicated by the occurrence of yellow spheres far away from the tip. This leads to an unexpected distribution of strain at later times, as demonstrated in Fig. 2a. Large shear strain concentrates at preferred distances to the origin (dashed circles), indicating a cascade-like structure. Similar cascade structures are observed in all components of strain. As an example, we show the distribution of normal strain $\varepsilon_{z z}$ in Fig. 2b. While blue spheres in the center reflect the compression induced by the tip, the pattern of blue and red spheres indicates a cascade of compression and dilation similar to that of the maximum shear strain (Fig. 2a). Obviously, this cascade structure is a characteristic property of the emergent plastic deformation; to show this, we compare the observed strain a
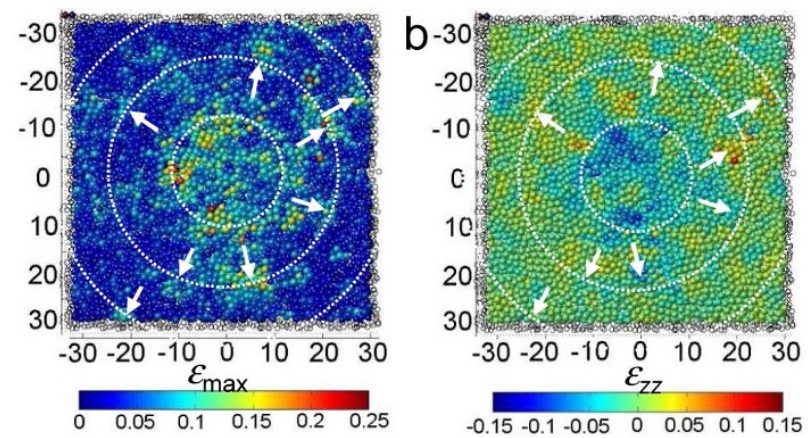

C

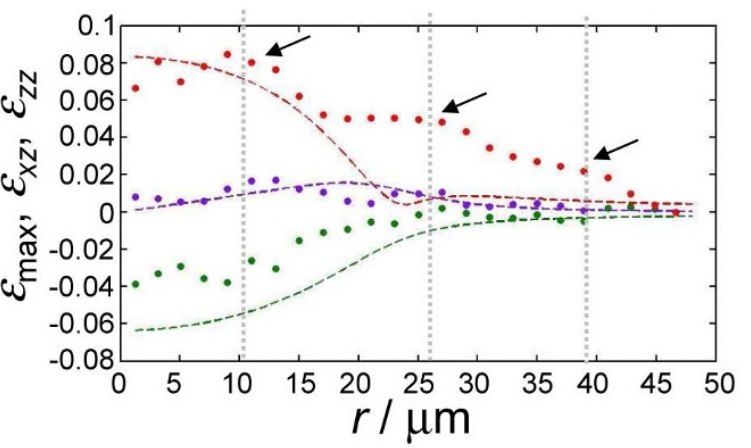

Figure $2 \mid$ Cascade structure of the incipient deformation. (a, b) Strain distribution in a $5 \mu \mathrm{m}$ thick section $10 \mu \mathrm{m}$ below the tip after $21 \mathrm{~min}$ of indentation. a, Distribution of the maximum shear strain, $\varepsilon_{\max }$.

b, Distribution of normal strain $\varepsilon_{z z}$. (c) Angle-averaged strain as a function of distance from the center of the tip. Symbols indicate the measured values of $\varepsilon_{\max }$ (red dots), $\varepsilon_{z z}$ (green dots), and the radial shear strain $\varepsilon_{\mathrm{rz}}$ (violet dots). Dashed lines indicate continuum predictions for $\varepsilon_{\max }, \varepsilon_{z z}$, and $\varepsilon_{\mathrm{rz}}$, respectively. Arrows indicate pronounced deviations from the predicted elastic distribution associated with the onset of permanent deformation. 

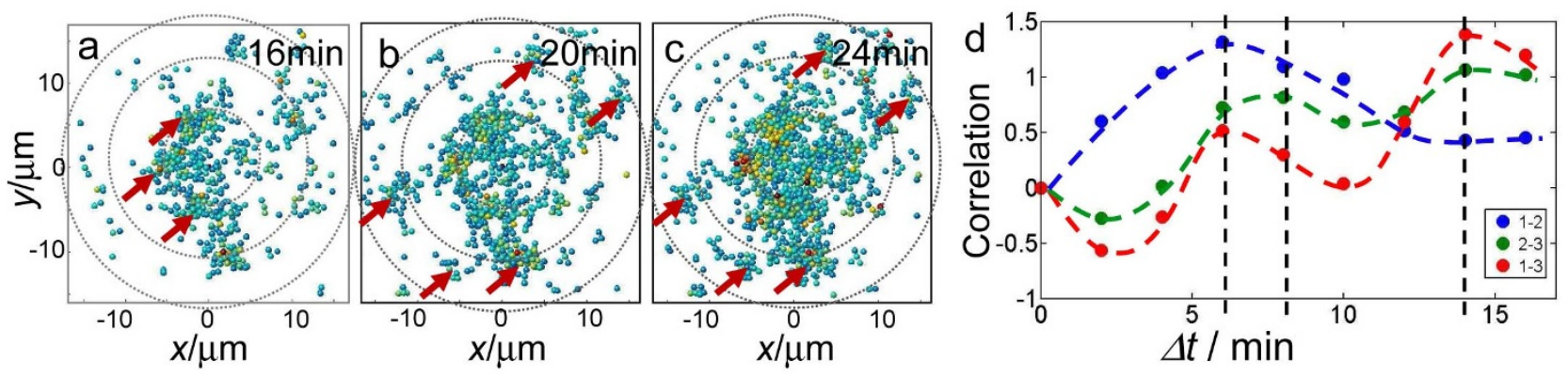

Figure $3 \mid$ Time evolution of strain. (a)-(c) Time series showing the evolution of regions of high strain after 16, 20, and 24 min of indentation. Red arrows indicate highly deformed zones penetrating the sample with time. (d) Time correlation of strain between the inner and middle (blue), middle and outer (red) and middle and outer (green) dashed circle in Figs. 3a-c. The time correlation is calculated by correlating the average strain within cylindrical shells of thickness $4 \mu \mathrm{m}$, and radii $R_{1}=10, R_{2}=25$, and $R_{3}=38 \mu \mathrm{m}$, centered at the origin. Maxima indicate the characteristic delay times of plasticity, $\Delta t_{12}=6, \Delta t_{23}=8$ and $\Delta t_{13}=14 \mathrm{~min}$.

distribution with the elastic strain distribution predicted by continuum elasticity. We assume the glass to be an isotropic elastic medium with Young's modulus, $E$, shear modulus, $\mu$, and Poisson ratio $v=0.34^{12}$. The elastic response of an indented elastic half-space has been treated analytically ${ }^{13,14}$; the elastic strain depends only on the radius of contact, $R_{c}$, between the indenter and the medium, and the maximum normal strain $\varepsilon_{0}$ in the center of contact. We measure $R_{\mathrm{c}}$ directly from the imaged contact surface between the tip and the colloidal glass and obtain $R_{\mathrm{c}}=22 \mu \mathrm{m}$. We then adjust $\varepsilon_{0}$ in order to best fit the experimental strain distributions, and obtain $\varepsilon_{0}=0.055$. The resultant elastic strain distributions are shown in Fig. 2c (dashed lines). Also shown by dots are the measured strain distributions. Striking differences occur at $R_{1}=10, R_{2}=25$ and $R_{3}=38 \mu \mathrm{m}$ (arrows) in all components of strain. These distances coincide precisely with the location of the circles in Fig. 2a and b; we associate these deviations from the elastic strain distribution with the onset of plastic relaxation.

Load-displacement curves of metallic glasses exhibit characteristic bursts at the onset of plastic flow that have been associated with the nucleation of shear bands, where slip occurs collectively along a surface. Such shear bands occur at an intermediate regime of indentation rates where the glass exhibits inhomogeneous flow ${ }^{8}$. The long time scales afforded by our colloidal glass permit us to access the regime of uniform flow at low indentation rates ${ }^{15}$ : Surprisingly, we observe a robust cascade structure of the incipient plastic deformation, demonstrating the occurrence of strong correlation in this uniform flow regime.

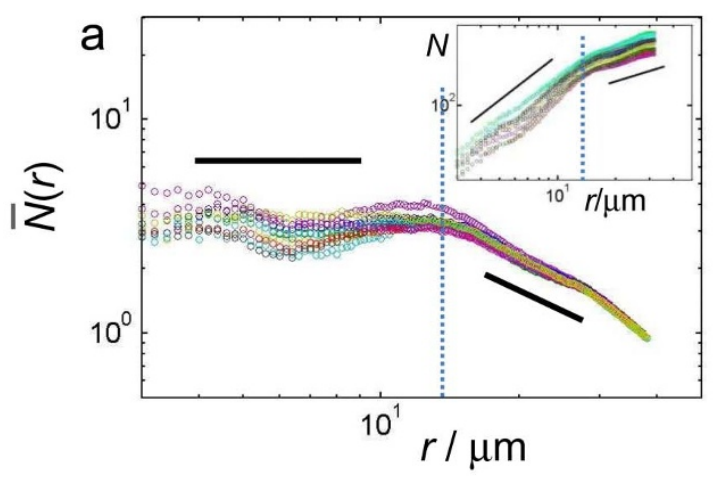

The long time scale of our colloidal glass allows us to even follow the evolution of this cascade in time: We focus on regions with large shear strain, and follow their time evolution in Fig. 3a-c. The incipient plastic deformation penetrates the glass with a characteristic propagation time. To determine this time scale, we measure the time delay between the plastic activity at the distances $R_{1}, R_{2}$ and $R_{3}$ in Fig. 2. We correlate the angle-averaged strain $\varepsilon_{i}(t)$ at $R_{i}$ at time $t$ with the strain $\varepsilon_{j}(t+\Delta t)$ at $R_{j}$ at some later time, $t+\Delta t$. The corresponding correlation functions, shown in Fig. 3d, reveal distinct time correlation: Maxima at $\Delta t=6,8$ and 14 min demarcate characteristic delay times associated with the propagation of plastic deformation, and reflect the time necessary to form new plastic zones in the strain field of existing ones. Thus, along with the spatial correlation, there is also strong time correlation in the incipient plastic deformation.

This strong spatial and temporal correlation results in a complex, highly correlated network of deformation. To elucidate it, we explore the analogy with fractal structures encountered in the aggregation of particles, where strong interparticle attraction leads to hierarchical structures characterized by a robust fractal dimension ${ }^{16}$. While for space-filling structures, the number $N(r)$ of particles within a distance $r$ grows as $N(r) \propto r^{D}$, where $D$ is the dimension of space, in open, fractal structures, $N(r) \propto r^{d_{\text {f }}}$, where $d_{f}<d$ is the fractal dimension. Surprisingly, we find that a similar description applies to the distribution of strain during the incipient deformation. To show this, we again focus on the high-strain particles shown in Fig. 3a-c, and determine their number $N(r)$ within a lateral distance $r$ to the origin. We plot $N$ as a function of $r$ in Fig. 4a, inset. Within the highly

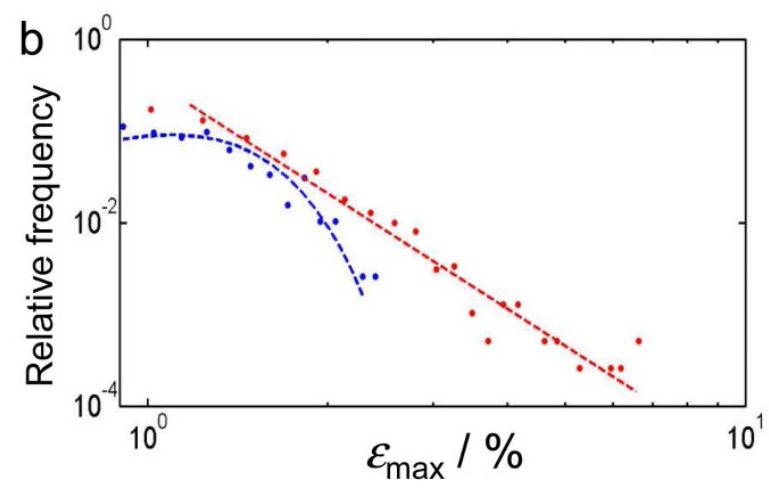

Figure 4 Fractal deformation field. (a) Scaling of the number $\bar{N}$ of high-strain particles, normalized by the homogeneous distribution, as a function of distance $r$ from the origin. Two regimes with different scaling are delineated by the vertical dashed line. For $r<15 \mu \mathrm{m}, \bar{N}$ is constant, indicating a compact, space-filling structure, while for $r>15 \mu \mathrm{m} \bar{N} \sim r^{-1.2}$ indicating a fractal structure. Colors indicate increasing time intervals of indentation, $t=15$ (red), 16 (violet), 17 (gray), 18 (green), 19 (blue), 20 (yellow) and $24 \mathrm{~min}$ (turquoise). Inset: Same data without normalization shows the fractal scaling of the number of high-strain particles according to $N(r) \sim r^{2}$, and $N(r) \sim r^{0.8}$. (b) Relative frequency of strain values as a function of strain magnitude for particles with distance $r<10 \mu \mathrm{m}$ (blue) and $r>20 \mu \mathrm{m}$ from the center (red). The dashed lines indicate fits to a Gaussian (red) and a power-law distribution (blue). 
deformed center, $N \sim r^{2}$, indicating a compact space-filling structure. Outside the central zone, however, $N$ grows slower, with a slope of $\sim 1.2$ indicating a fractal structure of deformation. To show this most clearly, we normalize by the homogeneous distribution, $\bar{N}=\pi \bar{\rho} r^{2}$, where $\bar{\rho}$ is the average density of high-strain particles, and show the normalized number of particles in Fig. 4a, main panel. The change from a horizontal to a negative slope of the data indicates the transition from a compact to a fractal structure of deformation. The excellent collapse of the data taken after different times of indentation demonstrates that this fractal structure is a robust property of the incipient deformation of the glass. This fractal structure of deformation allows plasticity to penetrate the glass at a low density. While our field of view limits the range of accessible length scales, we obtain further evidence of the fractal nature of deformation from the probability distribution of strain. We show histograms of strain values in Fig. $4 \mathrm{~b}$, where blue and red dots indicate particles in and outside the central zone, respectively. The data reveals the very different character of the distributions: while inside the central zone, particles exhibit a Gaussian strain distribution (blue dots and dashed line), indicating plasticity occurs in a statistically independent manner, outside the central zone, particles exhibit a power-law distribution (red dots and dashed line), indicating strong correlation, in agreement with the fractal structure of deformation. We hypothesize that due to the high shear stress in the center, plastic zones can form independently of each other, while at smaller stresses further away, plastic zones can only form when triggered by adjacent plastic zones, and hence plasticity occurs in a highly correlated fashion. These strong correlations result in a hierarchical structure of deformation.

\section{Discussion}

Recent theoretical work on disordered fiber networks ${ }^{9}$ indicates that the loss of network rigidity is associated with a true critical point. Our observation of a hierarchical strain distribution indicates that even a glass shows hallmarks of critical behavior at the loss of elasticity and the onset of plastic flow. Critical points demarcate qualitative changes of behavior, typically associated with symmetry changes. For the incipient deformation investigated here, the symmetry change occurs in time, and demarcates the transition from reversible elastic to irreversible plastic deformation. While the direct imaging of microscopic strain allows us to visualize its fractal structure in a colloidal glass, we expect our results to be general and apply to molecular glasses as well. We estimate from approximate conversion of the deformation rate from indentation to uniaxial strain rate that the strain rate of our experiment is $\sim 3 \times 10^{-5} \mathrm{~s}^{-1}$, a factor of three larger than the inverse structural relaxation time of our glass. This ratio is typically encountered in the high-temperature deformation of molecular glasses. For example, for typical strain rates employed in nanoindentation experiments of atomic glasses in the range of $10^{-3} \mathrm{~s}^{-1}$ to $1 \mathrm{~s}^{-1}$, the same ratio of imposed strain rate to relaxation rate is found in the range between 0.9 and 1.1 of the glass transition temperature. Deformation tests on metallic glasses show that indeed homogeneous deformation occurs at these strain rates and temperatures ${ }^{8}$, in agreement with our experiments. Therefore, our observations should be relevant for these atomic glasses as well.

The application of indentation on a colloidal glass reveals a surprising hierarchical structure of strain at the onset of permanent deformation. This hierarchical structure is reminiscent of a second-order transition, indicating that the glass at the onset of flow exhibits hallmarks of critical behavior, in agreement with recent theoretical work on fiber networks. The similarity between the fractal structure of deformation and that encountered in fractal aggregates of particles suggests an intriguing analogy between the mechanisms of structural arrest and shear-induced relaxation. Finally, the strong correlation of the incipient strain field should have important con- sequences for the deformation in small dimensions, such as the one exploited in applications of amorphous materials at small scales ${ }^{1}$.

\section{Methods}

We prepare a colloidal glass by sedimenting $1.55 \mu \mathrm{m}$ diameter silica particles onto a cover slip by centrifugation. The particles are suspended in a mixture of water and dimethylsulfoxide (DMSO) that matches their refractive index. The cover slip surface is coated with a $5 \mu \mathrm{m}$ thick layer of polydisperse colloidal particles to avoid boundary-induced crystallization. The resulting colloidal amorphous film has a volume fraction of $\phi \sim 0.60$, larger than that of the colloidal glass transition, $\phi_{\mathrm{g}} \sim 0.58^{17}$, and is therefore well inside the glassy state. A sewing needle attached to a piezoelectric translation stage is used to indent the colloidal glass. The needle has an almost hemispherical tip with radius $\sim 38 \mu \mathrm{m}$, and produces a strongly inhomogeneous stress field with a distinct maximum well below the contact surface. We lower the needle slowly at a rate of $2.9 \mu \mathrm{m} / \mathrm{h}$ into the amorphous sediment and use confocal microscopy to image individual particles in a $66 \mu \mathrm{m}$ by $66 \mu \mathrm{m}$ by $48 \mu \mathrm{m}$ section of the glass below the tip (Fig.1a). This technique allows us to follow the incipient plastic deformation directly at the particle scale. We determine individual particle positions in three dimensions with an accuracy of $0.03 \mu \mathrm{m}$ in the horizontal, and $0.05 \mu \mathrm{m}$ in the vertical direction ${ }^{18}$. We acquire image stacks every $60 \mathrm{sec}$ over a time interval of $25 \mathrm{~min}$ to track the motion of $\sim 3 \times 10^{4}$ particles in the imaged section. One image needs $45 \mathrm{sec}$ to acquire.

1. Kumar, G., Tang, H. X. \& Schroers, J. Nanomoulding with amorphous metals. Nature 457, 868-872 (2009).

2. Jang, D. \& Greer, J. R. Transition from a strong-yet-brittle to a stronger-andductile state by size reduction of metallic glasses. Nature Materials 9, 215-219 (2010).

3. Zhang, Y., Wang, W. H. \& Greer, A. L. Making metallic glasses plastic by control of residual stress. Nature Materials 5, 857-860 (2006).

4. Schuh, C. A. Nanoindentation studies of materials. Materials Today 9, 32-40 (2006).

5. Kim, J. -J., Choi, Y., Suresh, S. \& Argon, A. S. Nanocrystallization during nanoindentation of a bulk amorphous metal alloy at room temperature. Science 295, 654-657 (2002).

6. Li, J., Van Vliet, K. J., Zhu, T., Yip, S. \& Suresh, S. Atomistic mechanisms governing elastic limit and incipient plasticity in crystals. Nature 418, 307-310 (2002).

7. Schuh, C. A. \& Nieh, T. G. A nanoindentation study of serrated flow in bulk metallic glasses. Acta Mater. 51, 87-99 (2003).

8. Schuh, C. A., Lund, A. C. \& Nieh, T. G. New regime of homogeneous flow in the deformation map of metallic glasses: elevated temperature nanoindentation experiments and mechanistic modeling. Acta Mater. 52, 5879-5891 (2004).

9. Broedersz, C. P., Mao, X., Lubensky, T. C. \& MacKintosh, F. C. Criticality and isostaticity in fibre networks. Nature Physics 7, 983-988 (2011).

10. Falk, M. L. \& Langer, J. S. Dynamics of viscoplastic deformation in amorphous solids. Phys. Rev. E. 57, 7192-7205 (1998).

11. Bei, H., Lu, Z. P. \& George, E. P. Theoretical strength and the onset of plasticity in bulk metallic glasses investigated by nanoindentation with a spherical indenter. Phys. Rev. Lett. 93, 125504 (2004).

12. Schall, P., Weitz, D. A. \& Spaepen, F. Structural rearrangements that govern flow in colloidal glasses. Science 318, 1895-1899 (2007).

13. Hertz, H. Über die Berührung fester elastischer Körper. J. reine und ang. Math. 92, 156-171 (1881).

14. Huber, M. T. Zur Theorie der Berührung fester elastischer Körper. Ann. Phys. 319, 153-163 (1904).

15. Chikkadi, V., Wegdam, G., Bonn, D., Nienhuis, B. \& Schall, P. Long-Range Strain Correlations in Sheared Colloidal Glasses. Phys. Rev. Lett. 107, 198303 (2011).

16. Lin, M. Y., Lindsay, H. M., Weitz, D. A., Ball, R. C., Klein, R. \& Meakin, P. Universality in colloidal aggregation. Nature 339, 360-362 (1989).

17. van Megen, W., Mortensen, T. C., van Megen, W., Mortensen, T. C., Williams, S. R. \& Müller, J. Measurement of the self-intermediate scattering function of suspensions of hard spherical particles near the glass transition. Phys. Rev. E. 58, 6073-6085 (1998).

18. Weeks, E. R., Crocker, J. C., Levitt, A. C., Schofield, A. \& Weitz, D. A. Threedimensional direct imaging of structural relaxation near the colloidal glass transition. Science 287, 627-631 (2000).

\section{Acknowledgements}

This work is part of the research program of FOM (Stichting voor Fundamenteel Onderzoek der Materie), which is financially supported by NWO (Nederlandse Organisatie voor Wetenschappelijk Onderzoek). P.S. acknowledges financial support by a VIDI fellowship from NWO.

\section{Author contributions}

R.K. carried out all experiments presented in this manuscript. Y.R. performed the analysis of the data, and prepared the figures and wrote the first draft. D.D. did analysis for the figure 3 , 
also prepared this figure and corresponding part of the text. P.S. wrote the main manuscript text. All authors reviewed the manuscript.

\section{Additional information}

Supplementary information accompanies this paper at http://www.nature.com/ scientificreports

Competing financial interests: The authors declare no competing financial interests.
License: This work is licensed under a Creative Commons Attribution-NonCommercial-ShareAlike 3.0 Unported License. To view a copy of this license, visit http://creativecommons.org/licenses/by-nc-sa/3.0/

How to cite this article: Rahmani, Y., Koopman, R., Denisov, D. \& Schall, P. Probing incipient plasticity by indenting colloidal glasses. Sci. Rep. 2, 1064; DOI:10.1038/srep01064 (2013) 